\title{
Tuning Operators of Genetic Algorithms for Mesh Routers Placement Problem in Wireless Mesh Networks
}

\author{
Fatos Xhafa \\ Christian Sánchez \\ Admir Barolli \\ Technical University of Catalonia, Spain Technical University of Catalonia, Spain \\ E-Mail:fatos@lsi.upc.edu \\ E-Mail: csanchez@lsi.upc.edu \\ Seikei University, Japan \\ E-Mail: admir.barolli@gmail.com \\ Rozeta Miho \\ Leonard Barolli \\ Makoto Takizawa \\ Technical University of Tirana, Albania Fukuoka Institute of Technology, Japan \\ E-Mail:rmiho@fti.upt.al \\ E-Mail: barolli@fit.ac.jp \\ Seikei University, Japan \\ E-Mail: makoto.takizawa@computer.org
}

\begin{abstract}
Wireless Mesh Networks (WMNs) have become an important networking infrastructure for providing cost-efficient broadband wireless connectivity. WMNs are showing their applicability in deployment of medical, transport and surveillance applications in urban areas, metropolitan, neighbouring communities and municipal area networks. At the heart of WMNs are the issues of achieving network connectivity and stability as well as QoS in terms of user coverage. These issues are very closely related to the family of node placement problems in WMNs, such as mesh router nodes placement. As these problems are known to be computationally hard to solve, Genetic Algorithms (GAs) have been recently investigated as effective resolution methods. However, GAs require the user to provide values for a number of parameters and a set of genetic operators to achieve the best GA performance for the problem. The task of tuning parameters and operators is complex and, unfortunately, in most cases a specific GA configuration is needed for the problem under study to achieve high quality solutions. In this work we present the results of an experimental study for tuning the parameters and genetic operators of GA for mesh router nodes placement problem. The results of the study suggested a useful combination of parameter values and genetic operators for the problem, which could well serve as a reference for the family of node placement problems in WMNs solved through evolutionary algorithms.
\end{abstract}

\section{Introduction}

Wireless Mesh Networks (WMNs) [1] are important networking infrastructures. Such type of network is made up of wireless nodes, organized in a mesh topology, where mesh routers are interconnected by wireless links and provide Internet connectivity to mesh clients (a group of users).
WMNs distinguish for their low cost nature that makes them attractive for providing wireless Internet connectivity. Moreover, such infrastructure can be used to deploy community networks, metropolitan area networks, municipal and, corporative networks, and to support applications for urban areas, medical, transport and surveillance systems [2], [3].

At the heart of WMNs are the issues of achieving network connectivity and stability as well as QoS in terms of user coverage. These issues are very closely related to the family of node placement problems in WMNs [4], [5], [6], [7], [8], among them, the mesh router mesh nodes placement. Here, we consider the version of the the mesh router nodes placement problem in which we are given a grid area where to deploy a number of mesh router nodes and a number of mesh client nodes of fixed positions (of an arbitrary distribution) in the grid area. The objective is to find a location assignment for the mesh routers to the cells of the grid area that maximizes the network connectivity and client coverage. As node placement problems are known to be computationally hard to solve for most of the formulations [9], [10], [11], Genetic Algorithms (GAs) have been recently investigated as effective resolution methods. However, GAs require the user to provide values for a number of parameters and a set of genetic operators to achieve the best GA performance for the problem.

In this paper we present an experimental study for tuning the parameters and genetic operators of GA for mesh router nodes placement problem. The task of tuning parameters and operator is complex and, unfortunately, as shown in many studies in the GA literature [12], [13], [14], [15], [16], [17], [18], [19], in most cases a specific GA configuration is needed for the problem under study to achieve high quality solutions.

The experimental study aimed to identify the values of GA parameters and the combination of genetic operators that would yield the best performance of GAs for the problem. The objective was, on the one hand, to evaluate the performance of GA operators for different size instances of the 
problem. As genetic operators have different computational costs, it is important to evaluate how their performance might be affected by the size of the instance. On the other hand, due to characteristics of the problem at hand, namely, there are a number of mesh client nodes a priori distributed in the grid area, it was interesting to evaluate the performance of the genetic operators under different possible distributions (Uniform, Normal, Exponential and Weibull) of mesh client nodes in the grid area.

The rest of the paper is organized as follows. In Section 2 we briefly mention most common application scenarios of WMNs. The mesh router nodes placement problem is defined in Section 3. The Genetic Algorithm for the problem, and its main operators are presented in Section 4. The tuning of genetic operators is given in Section 5. We end the paper in Section 6 with some conclusions.

\section{Application scenarios of WMNs}

There are a number of application scenarios for which the use of WMNs is a very good alternative to offer connectivity at a low cost. It should also mentioned that there are applications of WMNs which are not supported directly by other types of wireless networks such as cellular networks, ad hoc networks, wireless sensor networks, standard IEEE 802.11 networks, etc.

Neighboring community networks. In a community, the usual solution is to deploy ADSL or cable. However, there are a number of limitations that WMNs can improve:

- A large percentage of areas between the houses could not receive wireless services.

- A broadband gateway between different houses could not be shared and wireless services should be established individually.

- A single path to each neighbor can communicate with the rest of neighbors or with the outside.

Corporative networks. This scenario corresponds to having a small network for an office or a medium sized network for all offices of a building or even a network to communicate offices located in different buildings. Other similar scenarios include airports, hotels, shopping centers, sports centers, etc.

Metropolitan area networks. Deploying WMNs in metropolitan areas has a number of advantages. The physical layer provides a higher average transmission to any cellular network and need not depend on a wiring. Also, deploying such infrastructure is much cheaper than cable or fiber and can be easily and rapidly deployed in areas with few resources, which have never had any network before.
Other scenarios. There are many more scenarios for which WMNs can be used. These include:

- Transportation systems: to provide information services to passengers, remote monitoring of vehicle safety and communications by the driver.

- Automatic control buildings: In buildings there are several electrical devices to be controlled, including light, elevator, air conditioning, etc.

- Medical and health systems: In a hospital information monitoring and diagnosis must be transmitted from one room to another.

- Surveillance: In corporate buildings, shopping malls and stores need broadband data transmission (images and videos basically) for monitoring and surveillance purposes.

\section{Mesh Router Node Placement Problem}

In this problem, we are given a grid area arranged in cells where to distribute a number of mesh router nodes and a number of mesh client nodes of fixed positions (of an arbitrary distribution) in the grid area. The objective is to find a location assignment for the mesh routers to the cells of the grid area that maximizes the network connectivity and client coverage. Network connectivity is measured by the size of the giant component of the resulting WMN graph (see later), while the user coverage is simply the number of mesh client nodes that fall within the radio coverage of at least one mesh router node.

An instance of the problem consists thus of:

- $N$ mesh router nodes, each having its own radio coverage, defining thus a vector of routers.

- An area $W \times H$ where to distribute $N$ mesh routers. Positions of mesh routers are not pre-determined, and are to be computed.

- $M$ client mesh nodes located in arbitrary points of the considered area, defining a matrix of clients.

It should be noted that network connectivity and user coverage are among most important metrics in WMNs and directly affect the network performance. Nonetheless, network connectivity is usually considered as more important than user coverage.

Notice from the above definition that mesh client nodes can be arbitrarily situated in the given area. For evaluation purposes, it is, however, interesting to consider concrete distributions of mesh client nodes such as Uniform, Normal, Exponential and Weibull distributions (the later has shown useful to model hotspots in university campuses.)

In fact, we can formalize an instance of the problem by constructing an adjacency matrix of the WMN graph, whose nodes are router nodes and client nodes and whose edges are links between nodes in the mesh network. Each mesh node in the graph is a triple $v=\langle x, y, r\rangle$ representing the $2 \mathrm{D}$ 
location point and $r$ is the radius of the transmission range. There is an arc between two nodes $u$ and $v$, if $v$ is within the transmission circular area of $u$. It should be noticed here that the deployment grid area is partitioned by cells, representing graph nodes, where we can locate mesh router nodes. We assume that in a cell, both a mesh router node and a mesh client node can be placed.

\section{Genetic Algorithms}

GAs have shown their usefulness for the resolution of many computationally hard combinatorial optimization problems. They are, of course, a strong candidate for efficiently solving mesh router nodes placement problem in WMNs. For the purpose of this work we have used the template given in Alg. 1.

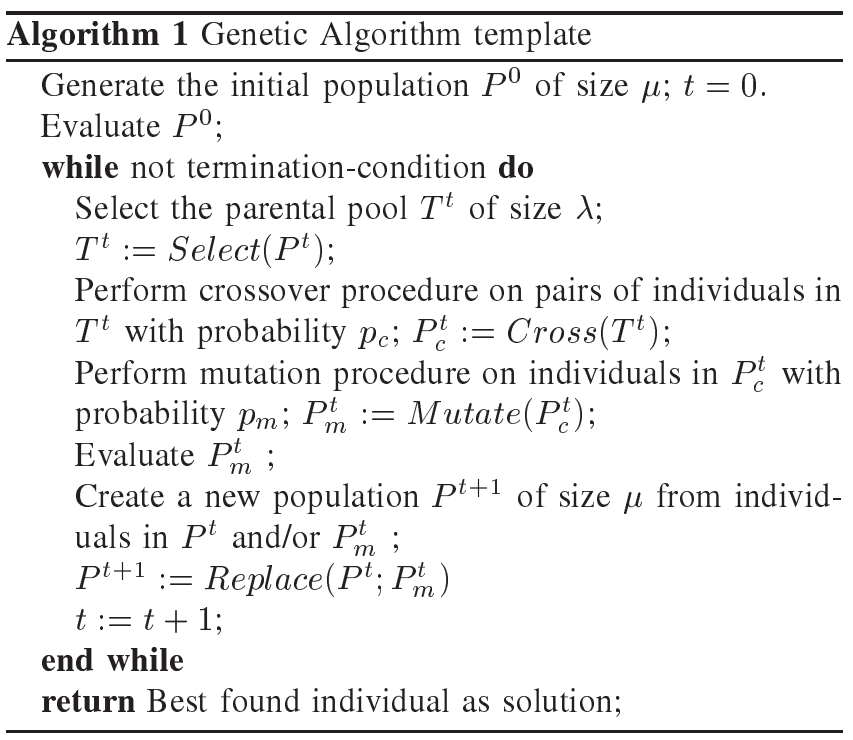

As can be seen from the template, several parameters intervene in the GAs: population size, intermediate population size, number of evolution steps, crossover probability, mutate probability and parameters for replacement strategies. On the other hand, there are the (families of) genetic operators: crossover operators, mutation operators, selection operators and replacement operators. As there are potentially large range values for parameters and different versions of operators, their tuning becomes crucial to the GA's performance.

\subsection{GA for mesh router node placement problem}

We specify below the particularization of GA template for the problem.

Encoding. The encoding of individuals (also known as chromosome encoding) is fundamental to the implementation of GAs in order to efficiently transmit the genetic information from parents to offsprings.
In the case of the mesh router nodes placement problem, a solution (individual of the population) contains the information on the current location of routers in the grid area as well as information on links to other mesh router nodes and mesh client nodes. This information is kept in data structures, namely, pos_routers for positions of mesh router nodes, routers_links for link information among routers and client_router_link for link information among routers and clients. Based on these data structures, the size of the giant component and the number of users covered are computed for the solution.

It should be also noted that routers are assumed to have different radio coverage, therefore to any router could be linked a number of clients and other routers. Obviously, whenever a router is moved to another cell of the grid area, the information on links to both other routers and clients must be computed again.

Fitness evaluation. The fitness function is of particular importance in GAs as it guides the search towards most promising areas of the solution space. Furthermore, in our case, we face an optimization problem with multiple criteria, including size of giant component, number of users covered, minimization of number of routers to deploy, etc.

In this work we will report results for the size of the giant component, although in our implementation we used a hierarchical approach that optimizes both the size of giant component and user coverage.

Selection operators. In the evolutionary computing literature we can find a variety of selection operators, which are in charge of selecting individuals for the pool mate. The operators considered in this work are those based on implicit fitness re-mapping technique. It should be noted that selection operators are generic ones and do not depend on the encoding of individuals.

Random Selection: This operator chooses the individuals uniformly at random. The problem is that a simple strategy does not consider even the fitness value of individuals and this may lead to a slow convergence of the algorithm.

Best Selection: This operator selects the individuals in the population having higher fitness value. The main drawback of this operator is that by always choosing the best fitted individuals of the population, the GA converges prematurely.

Linear Ranking Selection: This operator follows the strategy of selecting the individuals in the population with a probability directly proportional to its fitness value. This operator clearly benefits the selection of best endowed individuals, which have larger chances of being selected.

Exponential Ranking Selection: This operator is similar to Linear Ranking but now probabilities of ranked individuals are weighted according to an exponential distribution.

Tournament Selection: This operator selects the individuals based on the result of a tournament among individuals. 
Usually winning solutions are the ones of better fitness value but individuals of worse fitness value could be chosen as well, contributing thus to avoiding premature convergence. Particular cases of this operator are the Binary Tournament and $N$-Tournament Selection, for different values of $N$.

Crossover operators. The crossover operators are the most important ingredient of GAs. Indeed, by selecting individuals from the parental generation and interchanging their genes, new individuals (descendants) are obtained. The aim is to obtain descendants of better quality that will feed the next generation and enable the search to explore new regions of solution space not explored yet.

There exist many types of crossover operators explored in the evolutionary computing literature. It is very important to stress that crossover operators depend on the chromosome representation. This observation is especially important for the mesh router nodes problem, since in our case, instead of having strings we have a grid of nodes located in a certain positions. The crossover operator should thus take into account the specifics of mesh router nodes encoding. We have considered the following crossover operators, called intersection operators (denoted CrossRegion, hereafter), which take in input two individuals and produce in output two new individuals (see Alg. 2)

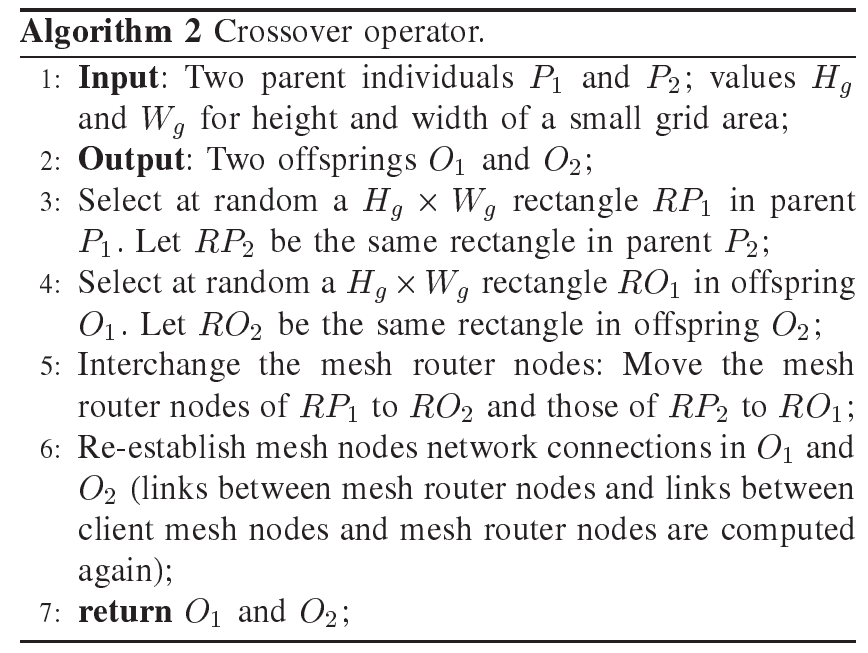

Mutation operators. The mutation operator is crucial for preventing the search from getting stuck in local optima by doing small local perturbations to the individuals of the population. Again, the definition of the mutation operators is specific to encoding of the individuals of the concrete problem under study. We defined thus several specific mutation operators as follows:

SingleMutate: Select a mesh router node in the grid area and move it to another cell of the grid area. After the move is done, network connections are computed again.
RectangleMutate This operator selects two "small" rectangles at random in the grid area, and swaps the mesh routers nodes in them. Certainly, in this case the modification of the individual is larger than in the case of SingleMutate.

SmallMutate This operator chooses randomly a router and moves it a small (a priori fixed) numbers of cells in one of the four directions: up, down, left or right in the grid.

SmallRectangleMutate This operator is similar to SmallMutate but now we select first at random a rectangle and then all routers inside the rectangle are moved with a small ( $a$ priori fixed) numbers of cells in one of the four directions: up, down, left or right in the grid.

Again, after the mutation is done, network connections (the links between routers and links between routers and users) are re-computed.

\section{Experimental Study}

The experimental study consisted in measuring the performance of the operators of the Genetic Algorithm, aiming to identify the combination of operators that would yield the best performance of GAs. The objective was, on the one hand, to evaluate the performance of GA operators for different size instances of the problem. It is clear that each genetic operator has its computational cost, and therefore it is important to evaluate how its performance might be affected by the size of the instance. For instance, RectangeMut ate is more computationally expensive than other operators. On the other hand, due to characteristics of the problem and hand, namely, there are a number of mesh client nodes a priori distributed in the grid area, it is interesting to evaluate the performance of the genetic operators under different possible distributions of mesh client nodes in the grid area.

Tuning process of genetic operators is complex, given the rather large number of operators and the possible synergies and conflicts among them. Moreover, the tuning has to be done in a way that the outcome of the study would be valid for any other instance of the problem. To this end, for the tuning process we have used randomly generated instances of three different grid area sizes $(32 \times 32,64 \times 64$ and $128 \times 128$, respectively). To avoid biased results, 15 independent runs of GA were performed and averaged results of giant component and user coverage are computed.

Additionally, the study involves many parameters of GAs and of the mesh node placement problem: initialization method, population size, intermediate population size, number of independent runs, number of evolution steps, cross probability, mutate probability, start choice, replace only if better and replace generational.

We exemplify below the tuning process of genetic operators for instances of $32 \times 32,64 \times 64$ and $128 \times 128$ grid area sizes. 


\subsection{Tuning of GA parameters}

Start choice. this parameter indicates the method used for generation of the first population. One important aspect here is to avoid fully random generation of the initial population to achieve its diversity at the very beginning of the search. To this end we considered ad hoc methods presented in [20] and StartNear method was used to generate one individual. In this method, mesh routers are concentrated in the central zone of the grid area. The rest of individuals are obtained from it using random perturbations.

Population size and intermediate population size. The relation between the two population sizes is set to (roughly) intermediate_population_size $=$ population_size $/ 2$. It should be remarked that with the increasing number of iterations, increases the number of connected routers as well (i.e. the size of the giant component). Certainly, the population size has an impact on how many iterations are needed to achieve full connectivity of mesh router nodes. The relationship between the population size and the size of the instance $N$ has followed the order $O\left(\log _{2} N^{k}\right)$, where $k$ is a correction factor parameter.

Number of evolution steps. This parameter indicates the number of generations to be performed by the GA algorithm. Clearly, a larger number of evolution steps would eventually provide a better solution but would take much longer execution time, while a small number of evolution steps might not suffice to converge GA to quality solutions. It is also to be taken into account that due to premature convergence, a large number of evolution steps would result in waste of execution time. In this study we have achieved good results with a value of number of evolution steps equal to $5 \cdot s i z e \_g r i d \_x \cdot k$, where size_grid_x denotes the number of cells along $x$ axis and $k$ represents a small correction factor.

Cross and mutate probabilities. As shown in GA literature, the influence of cross probability is much greater than that of mutation probability. Their respective values have been set to $p_{c}=0.8$ and $p_{m}=0.2$.

\subsection{Tuning genetic operators for instances of $32 \times 32$ grid area size}

The instances used in this case consisted of $32 \times 32$ grid area sizes, 48 mesh node clients positioned in the grid cells distributed according to normal distribution $N(\mu=15, \sigma=$ $32 / 10)$, and 16 routers are to be deployed in the grid area.

Mutation operators (32x32 grid area). We present in Table 1 the values of the parameters used in executing GA
Table 1. Parameter values used in GA executions for studying mutate operators for $32 \times 32$ grid area sizes.

\begin{tabular}{|l|c|}
\hline Parameter & Value \\
\hline nb_evolution_steps & 200 \\
\hline Cross_choice & CrossRegion \\
\hline Cross_probability & 0.8 \\
\hline population_size & 26 \\
\hline intermediate_population_size & 12 \\
\hline select_choice & SelectLinearRanking \\
\hline mutate_probability & 0.2 \\
\hline replace_generational & False \\
\hline replace_only_if_better & False \\
\hline Start_choice & StartNear \\
\hline
\end{tabular}

for studying the performance of GA when using different mutate operators.

The performance of the mutate operators is graphically shown in Fig. 1.

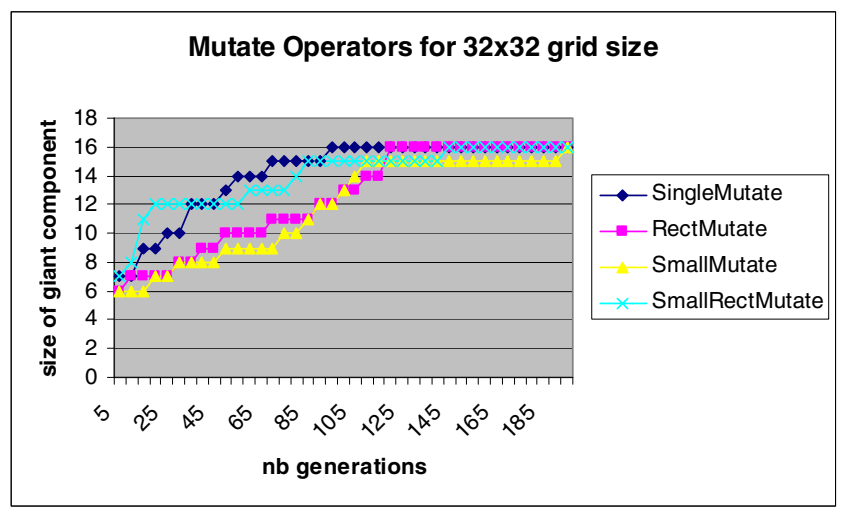

Figure 1. Performance of mutate operators in GA algorithm for $32 \times 32$ grid area sizes where 16 routers were to be placed and give coverage to 48 clients.

The mutation operator that is most convenient for instances of small size is the Sing leMut ate operator, which could be explained by the fact that this is less computationally costly as compared to other operators, which would spend unnecessarily more time for small size instances. This operator obtained best results in less generations than other operators.

Selection operators ( $32 \times 32$ grid area). In the case of the selection operators, the computational values were obtained using parameters in Table 2 used in executing GA.

The performance of the mutate operators is graphically shown in Fig. 2.

We observed that SelectExpRanking achieved better results among selection operators. At the other extreme are the operators that either converge very slowly due to not considering the fitness value to make the selection of individuals (this is the case SelectRandom) or those suffering from a 
Table 2. Parameter values used in GAs executions for studying select operators for $32 \times 32$ grid size.

\begin{tabular}{|l|c|}
\hline Parameter & Value \\
\hline Nb_evolution_steps & 200 \\
\hline cross_choice & CrossRegion \\
\hline cross_probability & 0.8 \\
\hline population_size & 26 \\
\hline intermediate_population_size & 12 \\
\hline mutate_choice & SingleMutate \\
\hline mutate_probability & 0.2 \\
\hline replace_generational & False \\
\hline replace_only_if_better & False \\
\hline start_choice & StartNear \\
\hline
\end{tabular}

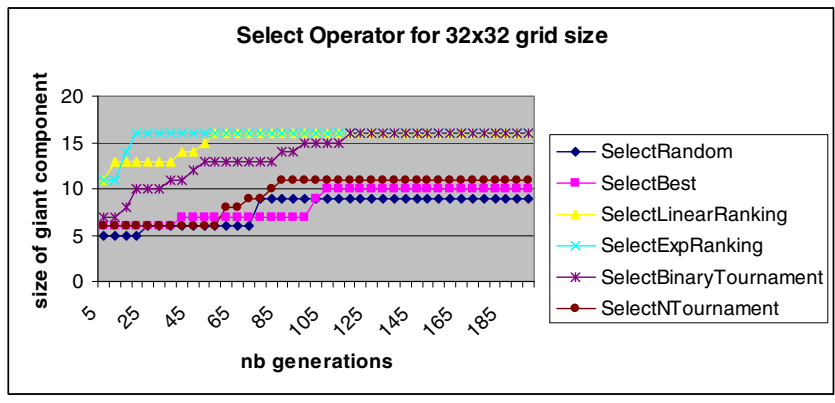

Figure 2. Performance of select operators in GA algorithm for $32 \times 32$ grid area sizes where 32 routers were to be placed and give coverage to 96 clients.

premature convergence due to selection of individuals (this is the case of SelectBest and SelectNTournament).

\subsection{Tuning genetic operators for instances of $64 \times 64$ grid area size}

The instances used in this case consisted of $64 \times 64$ grid size, 96 mesh node clients positioned in the grid cells distributed according to normal distribution $N(\mu=32, \sigma=$ $64 / 10$ ), and 32 routers are to be deployed in the grid area.

Mutation operators (64x64 grid area). We present in Table 3 the values of the parameters used in executing GA for studying mutate operators.

The performance of the mutate operators is graphically shown in Fig. 3.

For the instances of $64 \times 64$ grid area we observed that, again, SingleMutate outperforms the rest of mutate operators. Nonetheless, due to increase in size of the instances, RectMut ate and Small RectMutate improved considerably their performance.

Selection operators (64)64 grid area). In the case of the selection parameters, the computational values were obtained using parameters in Table 4 used in executing GA. The performance of the mutate operators is graphically shown in Fig. 4.
Table 3. Parameter values used in GA executions for studying mutate operators for $64 \times 64$ grid area sizes.

\begin{tabular}{|l|c|}
\hline Parameter & Value \\
\hline nb_evolution_steps & 400 \\
\hline Cross_choice & CrossRegion \\
\hline Cross_probability & 0.85 \\
\hline population_size & 36 \\
\hline intermediate_population_size & 17 \\
\hline select_choice & SelectLinearRanking \\
\hline mutate_probability & 0.15 \\
\hline replace_generational & False \\
\hline replace_only_if_better & False \\
\hline Start_choice & StartNear \\
\hline
\end{tabular}

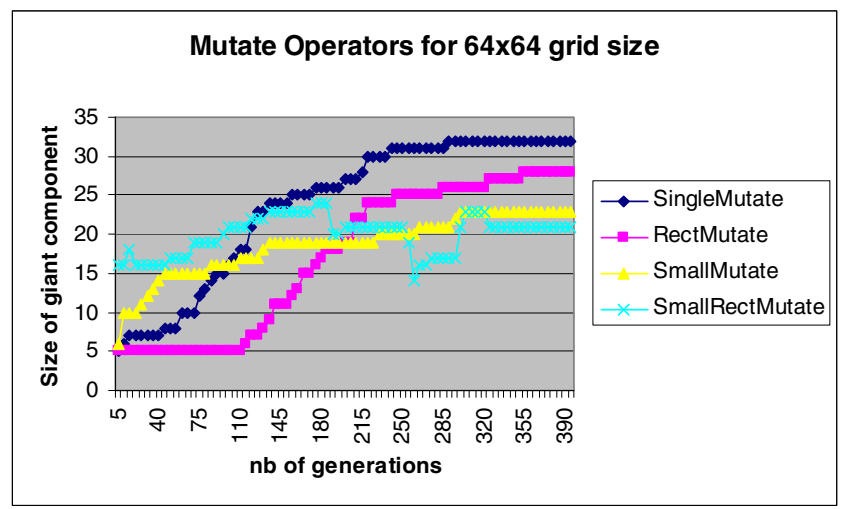

Figure 3. Performance of mutate operators in GA algorithm for $64 \times 64$ grid area sizes where 32 routers were to be placed and give coverage to 96 clients.

As can be seen, again SelectExpRanking achieved the best performance when compared to SelectlinearRanking, SelectExpRanking and SelectBinaryTournament due to a better ranking of individuals.

Table 4. Parameter values used in GA executions for studying select operators for $64 \times 64$ grid area size.

\begin{tabular}{|l|c|}
\hline Parameter & Value \\
\hline Nb_evolution_steps & 400 \\
\hline cross_choice & CrossRegion \\
\hline cross_probability & 0.85 \\
\hline population_size & 36 \\
\hline intermediate_population_size & 17 \\
\hline mutate_choice & SingleMutate \\
\hline mutate_probability & 0.15 \\
\hline replace_generational & False \\
\hline replace_only_if_better & False \\
\hline start_choice & StartNear \\
\hline
\end{tabular}




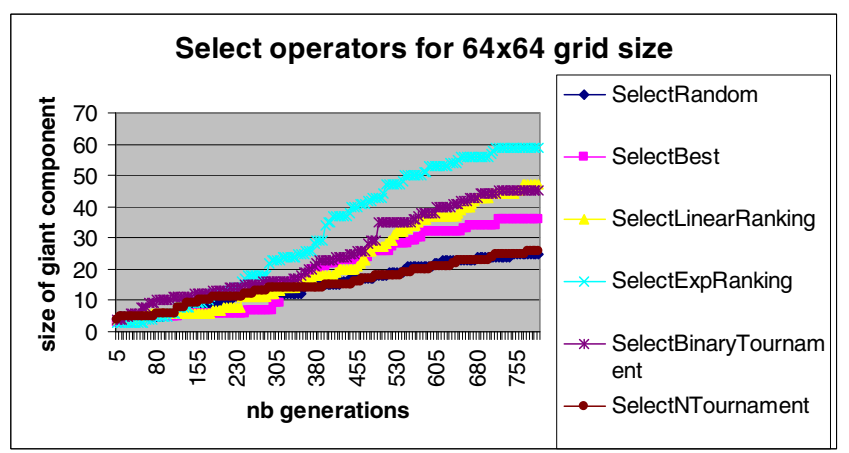

Figure 4. Performance of select operators in GA algorithm for $64 \times 64$ grid area sizes where 32 routers were to be placed and give coverage to 96 clients.

Table 5. Parameter values used in GA executions for studying mutate operators for $128 \times 128$ grid area size.

\begin{tabular}{|l|c|}
\hline Parameter & Value \\
\hline nb_evolution_steps & 800 \\
\hline Cross_choice & CrossRegion \\
\hline Cross_probability & 0.8 \\
\hline population_size & 49 \\
\hline intermediate_population_size & 24 \\
\hline select_choice & SelectLinearRanking \\
\hline mutate_probability & 0.2 \\
\hline replace_generational & False \\
\hline replace_only_if_better & False \\
\hline Start_choice & StartNear \\
\hline
\end{tabular}

\subsection{Tuning genetic operators for instances of $128 \times 128$ grid area size}

The instances used in this case consisted of $128 \times 128$ grid size, 192 mesh node clients positioned in the grid cells distributed according to normal distribution $N(\mu=64, \sigma=$ $128 / 10$ ), and 64 routers are to be deployed in the grid area.

Mutation operators (128x128 grid area). We present in Table 5 the values of the parameters used in executing GA for studying mutate operators.

The performance of the mutate operators is graphically shown in Fig. 5.

For this size it is observed that the operators based on moving several routers (within an imaginary small square) perform much better than the other operators. For larger sizes, therefore SingleMutate cannot compete with MutateRect and MutateSaml lRect mutation operators.

Selection operators (128x128 grid area). In the case of the selection parameters, the computational values were obtained using parameters in Table 6 used in executing GA.

The performance of the selection operators is graphically shown in Fig. 6.

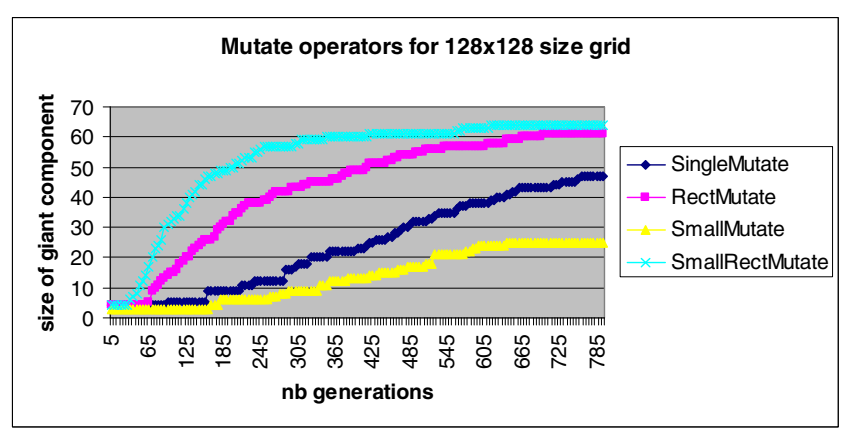

Figure 5. Performance of mutate operators in GA algorithm for $128 \times 128$ grid area sizes where 64 routers were to be placed and give coverage to 192 clients.

Table 6. Parameter values used in GA executions for studying select operators for $128 \times 128$ grid area size.

\begin{tabular}{|l|c|}
\hline Parameter & Value \\
\hline Nb_evolution_steps & 800 \\
\hline cross_choice & CrossRegion \\
\hline cross_probability & 0.8 \\
\hline population_size & 49 \\
\hline intermediate_population_size & 24 \\
\hline mutate_choice & SingleMutate \\
\hline mutate_probability & 0.2 \\
\hline replace_generational & False \\
\hline replace_only_if_better & False \\
\hline start_choice & StartNear \\
\hline
\end{tabular}

As seen in previous cases the operators that achieved a better ranking of individuals through the probability to best fitted individuals, showed better performance. As a matter of fact, SelectExpRanking achieved the best performance.

\section{Conclusions}

In this work we have presented an experimental study for tuning the parameters and genetic operators of Genetic

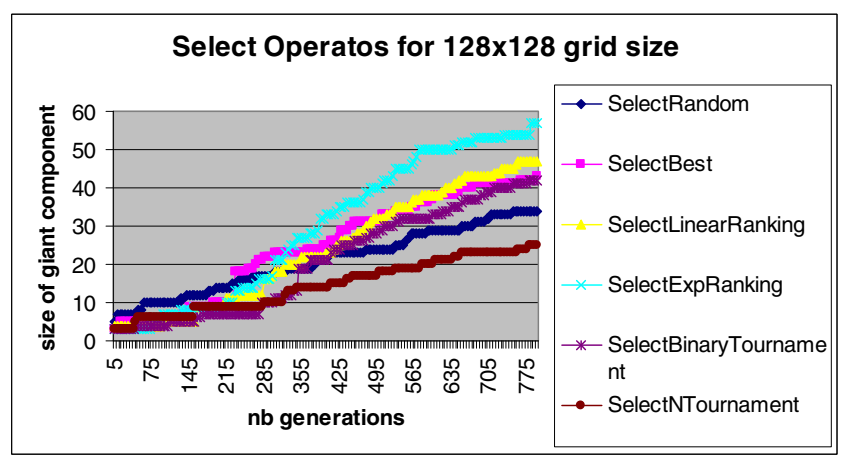

Figure 6. Performance of select operators in GA algorithm for $128 \times 128$ grid area sizes where 64 routers were to be placed and give coverage to 192 clients. 
Algorithms (GAs) for the problem of mesh router nodes placement in Wireless Mesh Networks (WMNs). Mesh router node placement is important to achieve network connectivity and stability and QoS in such networks. GAs are effective resolution methods for the problem, yet, using GAs requires the user to provide values for a number of parameters and a set of genetic operators to achieve the best performance for the problem. The results of the experimental study suggested a useful combination of parameter values and genetic operators for the problem, which we believe would well serve as a reference for the family of node placement problems in WMNs solved through evolutionary algorithms.

\section{References}

[1] I. F. Akyildiz, X. Wang, and W. Wang, "Wireless Mesh Networks: A Survey", Computer Networks, Vol. 47, No. 4, pp. 445-487, 2005.

[2] C. C. Chen and C. Chekuri, "Urban Wireless Mesh Network Planning: The Case of Directional Antennas", Technical Report No. UIUCDCS-R-2007-2874, Department of Computer Science, University of Illinois at Urbana-Champaign, June 2007.

[3] N. Nandiraju, D. Nandiraju, L. Santhanama, B. He, J. Wang, and D. Agrawal, "Wireless Mesh Networks: Current Challenges And Future Direction of Web-In-The-Sky", IEEE Wireless Communications, Vol. 14. No. 4, pp. 79-89, 2007.

[4] S. N. Muthaiah and C. Rosenberg, "Single Gateway Placement in Wireless Mesh Networks", Proc. of 8th IEEE International Symposium on Computer Networks, Available on line at: http://www.ece.uwaterloo.ca/ cath/iscn08.pdf, June 2008.

[5] P. Zhou, B. S. Manoj, and R. Rao, "A Gateway Placement Algorithm in Wireless Mesh Networks", Proc. of the 3-rd International Conference on Wireless Internet, Article No.: 1, October 2007.

[6] M. Tang, "Gateways Placement in Backbone Wireless Mesh Networks", International Journal of Communications, Network and System Sciences, Vol. 1, pp. 1-89, 2009.

[7] A. A. Franklin and C. S. R. Murthy, "Node Placement Algorithm for Deployment of Two-Tier Wireless Mesh Networks", Proc. of IEEE GLOBECOM-2007 pp. 4823-4827, November 2007.

[8] T. Vanhatupa, M. Hännikäinen and T.D. Hämäläinen, "Genetic Algorithm to Optimize Node Placement and Configuration for WLAN Planning", Proc. of 4-th International Symposium on Wireless Communication Systems (ISWCS-2007), pp. 612-616, 2007.

[9] M. R. Garey and D.S. Johnson, "Computers and Intractability -A Guide to the Theory of NP-Completeness", Freeman, San Francisco, 1979.

[10] A. Lim, B. Rodrigues, F. Wang and Zh. Xua, " $k$-Center Problems with Minimum Coverage", Theoretical Computer Science, Vol. 332, pp. 1-17, 2005.
[11] J. Wang, B. Xie, K. Cai and D.P. Agrawal, "Efficient Mesh Router Placement in Wireless Mesh Networks", Proc. of MASS-2007, pp. 1-9, October 2007.

[12] X. Yao, "An Empirical Study of Genetic Operators in Genetic Algorithms", Microprocessing and Microprogramming, Elsevier Science Publishers B. V., Vol. 38, Issues 1-5, pp. 707-714, 1993.

[13] J. Denzinger and J. Kidney, "Evaluating Different Genetic Operators in the Testing for Unwanted Emergent Behavior Using Evolutionary Learning of Behavior", Proc. of the IEEE/WIC/ACM International Conference on Intelligent Agent Technology, pp. 23-29, December 2006.

[14] M. O. Odetayo, "Empirical Study of the Interdependencies of Genetic Algorithm Parameters" Proc. of 23rd EUROMICRO'97, New Frontiers of Information Technology, pp.639, 1997.

[15] J. Tavares, N. Melab, E. G. Talbi, "An Empirical Study on the Influence of Genetic Operators for Molecular Docking Optimization", Technical Report, INRIA N6660, September 2008.

[16] F. Xhafa, B. Duran, A. Abraham, K. Dahal, "Tuning Struggle Strategy in Genetic Algorithms for Scheduling in Computational Grids", Neural Network World, Vol. 18, No. 3, pp. 209$225,2008$.

[17] F. Xhafa, L. Barolli, and A. Durresi, "An Experimental Study On Genetic Algorithms for Resource Allocation On Grid Systems", Journal of Interconnection Networks (JOIN), Vol. 8, No. 4, pp. 427-443, 2007.

[18] K. Hatta, S. Wakabayashi and T. Koide, "Adaptation of Genetic Operators and Parameters of a Genetic Algorithm Based on the Elite Degree of an Individual, Systems and Computers in Japan, Vol. 31, No. 1, pp. 29-37, 2001.

[19] K. Tahera, R. N. Ibrahim, and P. B. Lochert, "Adopting Dynamic Operators in a Genetic Algorithm", Proc. of the 9-th Annual Conference on Genetic and Evolutionary Computation (GECCO-2007), pp. 1533-1533, July 2007.

[20] F. Xhafa, C. Sanchez, L. Barolli, "Ad Hoc and Neighborhood Search Methods for Placement of Mesh Routers in Wireless Mesh Networks", Proc. of ICDCS Workshops of the IEEE 29th International Conference on Distributed Computing Systems (ICDCS-2009), pp. 400-405, 2009. 